\title{
Hereditary aspects of coronary heart disease
}

\author{
Frederick II. Epstein, M.D.* \\ Ann Arbor, Mich.
}

$\mathrm{A}^{\mathrm{m}}$ mong the factors which predispose to coronary atherosclerosis, heredity is usually accorded an important role. Thus, in a recent statement issued by a panel of experts on the factors which predispose to atherosclerosis, Katz and associates ${ }^{1}$ list these predisposing factors as: overweight, elevated blood cholesterol and blood pressure, excessive smoking, and heredity. When one considers the importance of the subject, it is surprising that there have been only relatively few reported studies concerned with familial aggregations of coronary heart disease. These suggest that coronary heart disease does, in fact, tend to aggregate in families, but the strength of these aggregations, as opposed to their mere presence, remains uncertain. Actually, modest concentrations of disease in families have been found for most common disorders for which they have been sought, as McKusick ${ }^{2}$ has pointed out. Such findings are not necessarily indicative of the operation of genetic factors, since families share their environment as well as their genes. It would seem timely to review the evidence for a familial predisposition toward coronary heart disease and to discuss the possible genetic mechanisms involved. Some of these problems have been briefly reviewed before, ${ }^{3}$ but no detailed analysis of current knowledge has been available, except in terms of disturbances of lipid metabolism, which represent but one facet of the total picture.

\section{Familial aggregations of coronary heart disease}

The frequent occurrence of coronary disease in family members of persons with xanthomatosis or high degrees of hypercholesterolemia has been known for many years. ${ }^{4}$ The extensive literature on this subject does not, however, contribute to the question whether, and to what extent, coronary heart disease, in the way it manifests itself as a major health problem in this and other countries, presents a familial pattern. Boas, Parets, and Adlersberg ${ }^{5}$ took a crucial step forward in suggesting that coronary disease in its usual form, particularly in young adults, might often be a similar but less extreme manifestation of familial hypercholesterolemia than the variety associated with xanthomatosis. In the intervening years, the view that coronary disease and cholesterol metabolism are interrelated has been greatly strengthened. The statement by Wilkinson and associates, ${ }^{6}$ on the basis of their studies of a large kindred in Michigan, that hypercholesterolemia per se-the "heterozygous state"-does not predispose toward coronary disease was not substantiated when we re-examined the same kindred 10 years later. ${ }^{4}$ Among general populations, many epidemiological

Presented in part at the Symposium on Genetics and Heart Disease, Philadelphia, Pa., January 24-26, 1963.

Received for publication July 12, 1963.

*Professor of Epidemiology, University of Michigan School of Public Health, Ann Arbor, Mich. Supported by Research Career Award HE-K6-6748 from the National Heart Institute, National Institutes of Health, United States Public Health Service. 
investigations have likewise demonstrated that the risk of having and of developing coronary disease increases with the level of serum cholesterol. ${ }^{7,8}$ Regardless of the importance of focusing major attention on the precursors of coronary disease, there is an unfortunate tendency at times to equate the genetic aspects of coronary heart disease, or for that matter atherosclerosis, with the genetic aspects of hypercholesterolemia. In a study of the hereditary aspects of coronary disease, a second point of departure should be the investigation of aggregations of the disease itself in kindreds of index cases representative of the population at large. Only through such an approach can the over-all importance of genetic factors in atherogenesis be assessed. At the same time, more is to be learned from investigations among special and selected groups, such as families with hypercholesterolemia, since these groups offer outstanding opportunities for research into intimate pathogenic mechanisms. These latter studies will not, however, yield an answer to the question whether genetic influences contribute significantly to the burden of these disorders as they present themselves in the general population. Therefore, a review of those studies which tend to provide tentative estimates of these influences will be presented. This review will make it apparent that there is a need for further and more extensive studies, and that, so far, there is inadequate evidence for taking a strong stand on whether genetic factors are or arc not of major importance in the development of the atherosclerotic diseases. In the making of this statement there is no attempt to belittle the significance of such factors. The attempt is to show the need for buttressing the evidence.

$A$. Studies among relatives of patients and controls. Most cardiologists have no doubt that they see not infrequently patients with coronary disease whose parents or siblings are similarly affected. ${ }^{9}$ At the same time, it would be important to know the extent to which such instances are representative of the total experience among all the patients who are seen over the years. Moreover, a disease which, in the United States, affects in a clinically demonstrable fashion as many as 5 per cent of middle-aged men ${ }^{10}$ and is the cause of death in about 40 per cent of middle aged men who die ${ }^{11}$ (not to mention older men) can be expected to coexist by chance in members of the same family with appreciable frequency. In addition the experience of cardiologists, hospitals, or clinics is not necessarily representative of events in the population at large. Finally, the diagnosis of coronary heart disease is by no means always simple nor is information on all family members readily obtained, or, if obtained, always reliable. It is easy, therefore, to find flaws in the data about to be reviewed but difficult to collect information which is better. In fact, the difficulties are such that the scarcity of accurate and representative data on familial occurrence of coronary disease is not surprising.

Gertler and White ${ }^{12}$ analyzed the family histories of 97 male patients who developed coronary heart disease prior to the age of 41 , and compared them with those of 146 male control subjects. It is important that absence of hypertension was a criterion for selection in the patients. Disease of the coronary arteries was twice as common a cause of death in the fathers of the patients as in the fathers of the control subjects ( 37 vs. 19 per cent); among the mothers, the corresponding trend was less marked (10 vs. 7.7 per cent). Nine per cent of the sibs of patients died of coronary disease, as opposed to 1 per cent of the sibs of controls. Gertler and White searched for multiple cases in the 100 sibships. There were 8 sibships with multiple cases of coronary disease; in 1 of the 8 there were 3 cases including the index cases, and in the other 7 there were 2 cases in the sibship. Gertler and White summarize this situation among siblings as follows: "The genealogies are interesting in that they fail to show a spectacular number of family members with coronary heart diseasc." This statement is of particular interest since it is becoming increasingly clear that familial aggregations need not be striking in order to be significant and meaningful. In fact, followup study on these patients suggests that the familial factor is, in fact, very important in these groups, ${ }^{13}$ and subsequent analysis of the original data indicates that a positive family history carries appreciable weight. ${ }^{14}$

Thomas and Cohen ${ }^{15}$ reported on the 
frequency of coronary heart disease, hypertension, obesity, and diabetes in parents, grandparents, uncles, and aunts of 266 consecutive medical students at Johns Hopkins University. ${ }^{15}$ The information, as in the study by Gertler and White, was based on carefully collected and reviewed medical histories. The major findings mav be summarized as follows: First, the frequency of coronary disease in the sons and daughters of the grandparents of the students was analyzed, representing the parents, uncles, and aunts of the students themselves. When both grandparents had coronary disease, 21.2 per cent of their sons were similarly affected when neither grandparent had the disease, only 4.1 per cent of the sons were reported to have the same condition. For matings of one affected and one unaffected grandparent, the frequency of coronary heart disease among their sons fell in between, being 8.2 per cent. Among the daughters of grandparents, the trend was in the same direction but less steep. The data may also be viewed in a different way: of 43 sons with coronary disease, 26 (60 per cent) were the offspring of grandparents of whom one or both were similarly affected. Next, the frequency of coronary disease among siblings of the students' parents was estimated. When the students' fathers were affected, his brothers showed a frequency of coronary disease of 15.8 per cent, about 4 times more than when the fathers were free from coronary disease. Coronary disease was also more frequent among the sisters of affected fathers. There were too few siblings of affected mothers to provide meaningful data. In general, data of this nature are, in part, conditioned by the age of the propositus; the older the propositus, the greater the chances of finding an affected relative.

Medical students at Johns Hopkins University cannot be considered to be a representative segment of the general population any more than were the patients or controls in Gertler and White's series, even though in the former case there was no deliberate exclusion of hypertensive subjects or limitation of age range. Nevertheless, the trend is similar in both studies, although one would not be justified in comparing the magnitude of the trend in view of methodological differences. Russek and Zohman ${ }^{16}$ also compared the frequency of a history of cardiovascular disease among the parents of 100 patients with coronary disease and that among the parents of 100 control subjects, without differentiating between the types of cardiovascular disease, as was done in Thomas' study; the frequency of a positive history was 67 per cent in one or both parents of patients, as compared with 40 per cent among the control subjects.

The most recent data were reported by Shanoff and associates, ${ }^{17}$ on the basis of a random sample of 102 patients and 100 controls drawn from patients who attended the Veterans Administration Hospital in Toronto. Thirty per cent of the patients but only 20 per cent of the controls had an affected father; a similar gradient was observed for mothers of patients and controls. The brothers of the patients were significantly more often affected than were the brothers of the controls; although the trend for their sisters was the same, the difference was not significant. It is of considerable interest that the disease became manifest in the sons on an average of 20 years earlier than in their fathers, which suggests that environmental changes over the past few decades may have tended to bring a genetic predisposition increasingly into the open.

$B$. Studies among special groups. It is well known that life insurance companies have long been aware of the significance of positive family histories. As an example, the experience of 27 companies on about 18,000 lives over a span of 15 years is cited, as reported by Lew. ${ }^{18}$ Persons insured at standard premium who report two or more cases of early cardiovascular-renal disease in their families have an excess risk of 75 per cent of dying of heart and circulatory diseases, and an excess risk of 80 per cent of dying specifically of coronary heart disease.

In many ways, studies on twins are ideally suited to establish the existence of genetic factors, although twins, even if reared apart, may still share etiologically significant environmental influences which may account in part for similarities between them. There are few such studies relating to coronary heart disease. Harvald and Hauge ${ }^{19}$ analyzed data on 3,100 pairs of twins in Denmark. Among 82 pairs over 
60 years of age the concordance rate for deaths from coronary occlusion was the same for monozygotic twins and dizygotic twins, whether of the same or opposite sex, which suggested to the authors that "genetic factors, generally speaking, play only a minor role in the etiology of coronary arteriosclerosis." However, Verschuer ${ }^{20}$ found a concordance rate of 19.0 per cent for "coronary sclerosis" among 21 pairs of monozygotic twins, as compared with a rate of 8.5 per cent in 47 pairs of dizygotic twins. Benedict ${ }^{21}$ reported on a pair of identical female twins who developed symptoms and signs of coronary insufficiency at about the same age in their early forties; blood pressure and total serum lipids and cholesterol were well within normal range in both twins. Lees and coworkers ${ }^{22}$ studied a pair of identical male twins, one of whom developed a myocardial infarction at the age of 27 ; detailed biochemical investigations suggested a defect in lipid metabolism.

The findings of Stare and his group on the frequency of coronary disease in Bostonians from Ireland and their brothers who stayed in the home country will be of much interest. ${ }^{23}$ Such investigations and others in progress will eventually help in segregating environmental from genetic factors in familial studies of coronary disease.

Scattered throughout the literature, one finds other data relative to familial aggregations of coronary heart disease on families with xanthomatosis or frank hypercholesterolemia. All these studies, in addition to those just reviewed, support the belief that coronary heart disease does, in fact, have a tendency to aggregate among blood relatives. From the point of view of segregating environmental from genetic factors, it would be of interest to have corresponding data on spouses, but these are lacking. In general, as Ciocco has reported, ${ }^{24}$ spouses tend to die of similar diseases, whether on account of sharing the same environment or associative mating.

\section{Mechanisms of genetic transmission}

A. Serum cholesterol and blood pressure levels. On the basis of clinical observation, it has been known for a long time that hypertension and hypercholesterolemia are frequently found among patients with coronary disease. One of the major, if not the major single contribution of epidemiological research in this area over the past 10 or 12 years has been the establishment of statistical proof for these observations in representative segments of the population and the accumulation of data which permit a quantitative as opposed to a qualitative estimate of these relationships. Thus, it can be calculated from the Framingham data ${ }^{25}$ that no less than about two thirds of the subsequent cases of coronary heart disease have cholesterol levels of 260 mg. per cent or over, blood pressure of 160 and/or $96 \mathrm{~mm}$. $\mathrm{Hg}$ or over, or a combination of the two. Since a genetic factor is involved in the control of both serum cholesterol and blood pressure levels, and since coronary disease is 3 or 4 times more common in middle-aged men when one or both of these factors are in the upper range than when they are not, it must inevitably follow that coronary disease will aggregate in families. A calculation based on a population model shows that two thirds of the aggregation of coronary disease in male siblings might be accounted for by the known familial trends in cholesterol and blood pressure levels. ${ }^{26}$

This important topic cannot be discussed without mention of the question whether blood pressure and cholesterol levels are determined by single genes or multiple genetic and environmental agencies. The estimate of genetic influences is, of course, much easier if only single genes are involved, and if the frequency of this gene in the population can be determined. There seems to be little doubt at this stage that it is extremely difficult, if not impossible, to demonstrate bimodality in the distribution of either blood pressure or cholesterol. ${ }^{3,4}$ This, in itself, is no proof that bimodality might not be hidden within these skewed distributions. In fact, Cicchinelli $^{27}$ has derived a procedure, based on the method of maximum likelihood, which permits, with the aid of a computer, the dissection of any skewed curve into two separate Gaussian distributions, and he has applied this procedure to blood pressure data from two epidemiological studies. While the biologic, as opposed to the mathematical, significance of this work 
awaits confirmation, it opens a new approach to the study of this problem. For the time being, from a purely practical point of view, it must be accepted that it is not possible to indicate the degree of probability of any given blood pressure or cholesterol level to be within the normal or abnormal distribution, if such does, indeed, exist.

A preliminary view of our own data from the Tecumseh study to be described later tends to support the belief that both serum cholesterol and blood pressure levels are determined over the whole range of the distribution by multiple genetic and environmental factors. ${ }^{28}$ These findings are in accordance with the data of Miall and Oldham $^{29}$ on blood pressure correlations in propositi and their first-degree relatives, and the data of Schaefer, Adlersberg and Steinberg ${ }^{30}$ and our own previous reports ${ }^{4}$ in regard to serum cholesterol levels. In our recent studies, ${ }^{28}$ serum cholesterol and blood pressure levels in parents and children in the town of Tecumseh were plotted by sex and 10-year age groups in parents and 5-year age groups in children, using a computer which calculated means, intercepts, slopes, and correlation coefficients at the same time. A summary of the correlation coefficients for cholesterol values in parents and their children (fathers vs. sons, fathers vs. daughters, mothers vs. sons, mothers vs. daughters) indicates that 31 of the 39 correlations were based on 50 or more observations. Twenty-three of these 31 coefficients were significantly different from zero at the 5 per cent or 1 per cent level. Although the correlations are low, generally of the order of about 0.2 , it is noteworthy that most of the slopes of the regression lines had a positive value, indicating a regular although not strong tendency for parents and children to resemble each other over the whole range of the distribution rather than just in the upper range, since their regressions could be shown to be essentially linear. For systolic blood pressure, a similar picture prevailed, although the correlation coefficients tended to be somewhat lower than those for cholesterol. Of 52 correlation coefficients, 35 were based on 50 or more observations. Nineteen of these 35 correlation coefficients were significantly different from zero. Again, most of the slopes were positive and the regressions essentially linear, leading to the same conclusions which were drawn with regard to comparisons of cholesterol levels.

There has, of course, never been any question that genetic factors are important in determining blood pressure and serum cholesterol levels, a view also supported by several studies on twins. The problem relates to the relative importance of genetic and environmental factors not only in the upper range but over the whole distribution of these variables. There is no answer to this question at this time. However, there is, at least, some evidence that familial aggregations of coronary heart disease are in part conditioned by these two factors.

With regard to blood pressure, only the studies by Thomas and Cohen, ${ }^{15}$ already cited, provide relevant information in this connection. When one or both parents (i.e., the parents of the medical students under study) were hypertensive, the frequency of coronary disease in their siblings was 5 per cent, a little but not significantly higher than the frequency of 4.2 per cent when both parents were normotensive. Similarly, the frequency of coronary disease in the offspring (sons and daughters combined) of the students' grandparents was slightly but not significantly higher when one of the grandparents was hypertensive; however, the daughters of hypertensive parents had significantly more coronary disease than did those of normotensive parents. In view of the diagnostic difficulties inherent in these data, the somewhat surprising lack of a stronger effect may not be meaningful.

With regard to serum cholesterol levels, the evidence is somewhat more telling. Thomas, ${ }^{31}$ again, noted that no less than 9 per cent of 612 students had serum cholesterol levels of $300 \mathrm{mg}$. per cent or overa startling figure in itself. Thirty-two per cent of these students had a parent with coronary disease, as opposed to 12 per cent of students with levels below $300 \mathrm{mg}$. per cent. It may be assumed that some of the parents of the hypercholesterolemic students were hypercholesterolemic themselves. More recently, Basset ${ }^{32}$ studied 19 male medical students and one resident 
physician who had fathers with coronary disease, and two groups of males who served as controls. The average cholesterol level in the subjects was $219 \mathrm{mg}$. per cent, as compared with 181 and $193 \mathrm{mg}$. per cent, respectively, in the two control groups. Gofman's extensive data on 876 employed men, 30 to 39 years of age, point in the same direction. ${ }^{33}$ Using the atherogenic index derived from ultracentrifugally determined beta-lipoprotein levels at different flotation rates, and setting at 1.0 the relative risk of having a father dead of heart disease when the atherogenic index was under 40 units, Gofman found that the relative risk increased steadily to a value of 4.87 when the index was 100 units or over. It is of interest that only 3 per cent of the men had indices under 40 , whereas 46 per cent had indices over 70 , at which point the relative risk had reached the level of 3.28 .

B. Other mechanisms of genetic transmission. It is most likely that blood pressure and serum cholesterol are not the only factors which may predispose to aggregations of coronary disease in families. Gertler and White, ${ }^{12}$ Pell and d'Alonzo, ${ }^{34}$ and Brontë-Stewart ${ }^{35,36}$ have all studied the relation of blood groups to coronary disease. Only Brontë-Stewart and his colleagues found a significant relation, indicating a deficiency of blood group $\mathrm{O}$ individuals among patients with coronary disease manifested as myocardial infarction but not among patients presenting with angina pectoris. Interestingly, this phenomenon was demonstrable only in the ethnic subdivisions which showed a relatively low prevalence of coronary heart disease. Brontë-Stewart believes that the genetic factor involved may be masked among populations with a high prevalence of disease, since, in them, environmental factors, such as diet, could overshadow in importance the genetic element related to blood groups. Although the relationship between blood groups and coronary disease remains in doubt, such a masking effect might explain in part the negative results obtained in the other two studies.

Other hereditary traits might well enter into a predisposition to coronary atheroma. Thus, Murphy and Mustard ${ }^{37}$ found platelet survival and turnover short- ened and in vitro clotting tests more active in persons with atherosclerotic disease, or a history thereof, than in controls. The anatomic configuration of the coronary tree, presumably an inherited trait, might influence the deposition of atheromata on account of the alterations in the dynamics of blood flow. Tissue repair responses to injury may also be under hereditary control. Body build and obesity, likewise influenced by heredity, are related to predispositions to coronary disease, as may be certain emotional characteristics, which could be, to some extent at least, inborn. There is clearly no end to the possibilities for idle speculation; the need is for factual information.

In view of the recent interest in the relation between elevated serum triglycerides and coronary disease, it is recalled that Hirschhorn $^{38}$ has found hyperlipemia to occur with a frequency of as much as 2 or 3 per cent among medical students. Although the relationship between triglyceride and cholesterol metabolism or their precise genetic determinants have not been established, these problems are of certain relevance to atherogenesis and its hereditary aspects.

In a discussion of the mechanisms of genetic transmission, the frequency of coronary disease in diabetic patients must arouse curiosity. It is rather strange how little work has been done on the simultaneous study of these two disorders in the same families, and that reports from the major epidemiological studies of coronary heart disease have so far omitted data on the coexistence of these conditions. Boas ${ }^{39}$ was surprised to find that the age of onset of coronary disease was the same in his diabetic and nondiabetic patients. He suggested that coronary disease might not be so much a consequence of diabetes, but that both could be due to a similar, underlying disturbance, manifesting in some as diabetes, in others as coronary disease. Conn and Fajans ${ }^{40}$ suggested that the defect in protein synthesis in diabetic patients which might cause the elaboration of an abnormal insulin molecule could also be responsible for the structural faults in the vascular systems of these persons. As part of our epidemiological study of Italian and Jewish clothing workers in New York 
some years ago, ${ }^{41}$ we attempted to collect some data on these interrelations. ${ }^{42}$ Family histories on siblings of 106 probands with diabetes, and siblings of 224 probands with coronary heart disease were obtained. Coronary disease was reported for 9.8 per cent of the 173 male and 4.9 per cent of the 144 female siblings of probands with diabetes; the corresponding figures for the 318 male and 309 female siblings of probands with coronary heart disease were 21.1 and 10.0 per cent, respectively. Thus, coronary heart disease was found more frequently among the siblings of probands with coronary heart disease than among the siblings of probands with diabetes. Conversely, diabetes was reported more frequently for siblings of diabetic probands than for siblings of probands with coronary disease; 11.6 per cent of the male siblings and $\mathbf{1 2 . 5}$ per cent of the female siblings of diabetic probands also had diabetes, whereas diabetes was reported for only 2.5 per cent of the male siblings and 3.9 per cent of the female siblings of probands with coronary disease. Coexistent diabetes and coronary disease was reported for 2.3 per cent of the male siblings and 1.4 per cent of the female siblings of diabetic probands; the corresponding figures for siblings of coronary disease probands were 2.5 and 1.3 per cent, respectively. Thus, coronary disease was found more frequently among the siblings of probands with coronary disease than among the siblings of probands with diabetes; conversely, diabetes was found more often among the siblings of probands with diabetes than among the siblings of probands with coronary heart disease. Calculations to demonstrate associations between coronary disease and diabetes showed no deviations from randomness, i.e., there was no evidence for an association between these disorders. These results were disappointing, but it must be remembered that the data were all based on histories obtained from the proband. It is quite possible that a person with a given disese is more apt to report the same disorder in a sibling than is a person who does not have the disease himself. Many of the studies already discussed suffer from the same limitations. We reviewed, some years later, the charts of all patients with diabetes who registered at the University Hospital in
Ann Arbor between 1951 and 1956, and whose age at first registration was between 20 and 40 years. $^{43}$ Thus, the index cases were all diabetics whose disease started relatively early and who might be expected to show a stronger genetic component among their families than older diabetics. Of 558 such patients, 107 lived within a 30-mile radius of Ann Arbor. Thirty-one of these patients were interviewed in regard to their family history, whereas the history on the other 76 patients was obtained from their charts. This review likewise failed to reveal any striking concentrations of coronary discase in parents and siblings of diabetics. The actual rates for the occurrence of coronary disease in these parents and siblings of diabetics were strikingly similar to those reported by Thomas, ${ }^{15}$ who found coronary disease about 1.5 times as frequently in siblings of diabetics as in nondiabetics; this difference was not statistically significant. Evidence that coronary disease and diabetes might be different facets of a similar disturbance is, therefore, lacking, but proof of such a relationship is so difficult to obtain and the hypothesis is so attractive that it should be pursued further.

\section{Methodological problems}

The study of familial factors in health and disease hinges on a number of requirements. It is necessary to obtain groups of patients and controls who are representative of the universe from which they are derived. Data on a major proportion of the kindreds involved must be available. The data must be accurate. In situations in which familial aggregations are not striking, the population studied must generally be large. If, after all these provisions have been fulfilled, familial aggregations are found, the relative importance of genetic and environmental factors must be evaluated. In the case of coronary disease, all of these requirements present methodological problems. Most serious, perhaps, are the difficulties of diagnosis, particularly of preclinical disease. It is difficult enough to diagnose coronary disease on many occasions even when the subject is available for careful clinical examination, but it seems mcre than risky to make the diagnosis in a relative, deceased or beyond 
reach, on hearsay evidence; yet, this is what one is often forced to do in these studies.

Before we illustrate these problems by describing some of the results from an actual study, a brief look at the problems of general approach and numbers may be in order. Let us assume, for the sake of simplicity, that every middle-aged man in a representative American population has, on an average, one brother, so that the total population of middle-aged men would be represented by a large number of pairs of brothers. Let us take at random 1,000 such pairs and postulate, in fair agreement with observation, that the prevalence of coronary heart disease in such a population is 5.5 per cent. Among the 2,000 brothers in this population, there will be 110 cases of coronary disease, 55 occurring in the first and 55 in the second member of the pair. On the basis of chance alone, 6 of these cases will occur in 3 brother-pairs ( 0.0055 times 0.0055 equals $0.00302)$; the other 104 cases will be "singleturns." If there were familial aggregations of coronary disease equal to 2 or 4 times random expectation, 6 or 12 brotherpairs, respectively, would be affected simultaneously. It is noteworthy that even with a risk 4 times greater than chance, "familial aggregations," as it were, will be observable in only 12 out of 1,000 sibships.

These findings may be considered either from the "index case-control" or "population genetics" point of view. Of the two, the index case-control approach is more commonly employed and, in some ways, is more easily accomplished in practice. In the situation in which familial aggregations are 4 times greater than chance it will show that the prevalence in sibs of propositi is 22 per cent $(12 / 55)$, in sibs of controls 4.6 per cent $[(55-12) /(1,000-55)]$, indicating that the disease is 4.8 times as common in sibs of index cases as in controls. The disadvantage of this approach is its failure, in general, to reveal the proportion of cases in a population which are, in fact, familial. Therefore, in the situation in which a total population is studied, one will obtain the additional data that, in the same example, 22 per cent of the cases $(24 / 110)$ were "familial." Up to this point, the information is, of course, purely statistical, and further data are needed to indicate the genetic and environmental attributes which cause these aggregations. It is of interest that these calculations based on this useful model are highly influenced by prevalence rates. A prevalence rate of 5.5 per cent for coronary disease reflects, of course, no more than the "top of the iceberg," an additional number of cases being hidden beneath the surface on account of the relative insensitivity of diagnostic instruments. With a prevalence rate of 20 per cent, which is closer to the objective picture as a pathologist would see it, the corresponding calculations would show a sixteen-fold differential for the index casecontrol approach and no less than 80 per cent of "familial aggregation," assuming again a concordance rate of affected sibs 4 times greater than chance. The argument will not be pursued beyond this point but may help to illustrate the complexities of the issues at stake and the need for studies to test the actual facts against these population models.

In our own prospective studies in the town of Tecumseh, Michigan, ${ }^{44-46}$ we have an opportunity to carry out one such test among a series of other integrated activities.* Of the 9,600 inhabitants of this town, approximately 90 per cent have been examined once. The preliminary data to be reported are based on these examinations. More reliable information will emerge with each subsequent round of re-examinations. During the first round, 248 persons with clinically manifest coronary heart disease were identified, using criteria serving the needs of this particular analysis. These belonged to 242 sibships, since there were 6 sibships with multiple cases. The sibships comprised 1,213 men and women. About a quarter of these were examined, and about another quarter were deceased. A third of the sibs lived beyond immediate reach, but about 1 in 8 lived sufficiently close to the study area to be interviewed; the latter persons have not yet received a physical examination. This is one of the hazards of doing such studies in the United

\footnotetext{
*These investigations were conducted in collaboration with the Research Staff of the Tecumseh Cummunity Health Study, Cardiovascular Research Center, University of Michigan, supported by Program Project Grant H-6378 from the National Heart Institute, National Institutes of Health, U. S. Public Health Service.
} 
States, where the population is relatively mobile. It is left open to question whether living within the study area or farther away is related to the disease under investigation and might thus introduce a bias. In this essentially total community comprising, in addition to younger people, 2,214 men and women who were 40 years of age and over, there were 6 sibships, as already mentioned, in which there was more than a single case of coronary heart disease among the members examined; in one of these, in addition to the 2 index cases, a sister died of a heart attack at the age of 52, and in another, 2 additional brothers and a sister were interviewed and one of the interviewed brothers had suspect coronary disease. These pedigrees illustrate the kind of data obtainable in a large epidemiological study of this type. The multiple cases in 2 of these 6 sibships are half-brothers and half-sisters.

In addition to these 6 sibships, there were 10 other sibships in which there was one examined person with coronary disease and one interviewed sibling who gave a history of myocardial infarction or angina pectoris; in one of these 10 sibships, 2 additional brothers died of "heart trouble." Another category includes 43 sibships in which, in addition to the examined index case, a brother or sister had died of a "heart attack" or "heart trouble"; these sibships included no interviewed sibling who reported evidence of coronary disease. There remained 183 sibships, comprising 843 persons, in which the index case was the only known instance of "probable" coronary heart disease in the sibship, and in which no person was reported to have died of this disease. In these sibships, of course, as well as in the others, new events of coronary heart disease will occur as time progresses.

Disregarding for the time being the problems of diagnostic identification already mentioned, it may now be asked whether the familial data just described indicate that coronary heart disease aggregates in these sibships more often than expected by chance. The answer to this question involves comparison with control kindreds or calculation of expected versus observed frequencies, utilizing the data from the total population, as discussed elsewhere. ${ }^{26}$ This intricate analysis has not yet been completed. In the meantime, it would seem fair to state that a clinical rather than statistical look at these data fails to indicate a striking degree of familial concentration of cases on the basis of a one-time cross-sectional survey. This is similar to the situation observed in other chronic diseases. This may be due, in part, to the fact that in conditions which are influenced by multiple genes a large part of the variation between individuals is concealed ${ }^{47}$; on the other hand, even slight differences in disease frequency between relatives of probands and controls may be very meaningful. ${ }^{48}$ Thus, it cannot be concluded in any way that familial factors are of relatively minor importance in the genesis of coronary heart disease. It seems more likely that the error lies in using as an index of genetic predisposition the end result, i.e., clinically manifest disease, rather than the underlying biologic disturbances in terms of metabolic or other defects. If one could identify and measure all of these predisposing traits, it would probably emerge that they are even more widespread than the prevalence of the disease would suggest and show more clear-cut distributions within kindreds. Prevention of coronary heart disease demands that the carriers of these traits be identified so that prophylactic measures can be instituted at an early age among genetically susceptible individuals. Although there is undoubtedly very much more to be learned about these traits, the association between coronary disease and elevated levels of serum cholesterol and blood pressure makes it likely that the determinants of these particular two variables are, for lack of a better term, an integral part of an "atherosclerotic constitution." In fact, the data from the Tecumseh Study indicate that elevation of one or both of these variables (serum cholesterol $260 \mathrm{mg}$. per cent or above, and/or blood pressure $160 \mathrm{~mm}$. Hg systolic and/or $96 \mathrm{~mm} . \mathrm{Hg}$ diastolic or above) occurred among 7 of the 12 index cases in the 6 sibships in which there was more than one affected and examined individual. Moreover, in 5 of these 6 sibships, there was at least one person with at least one of these two variables in the elevated range. In sibships with one examined and one interviewed person with coronary disease, 7 of the 10 index cases showed elevation of one 
or both of these variables. Altogether, rather more than one half of the persons with coronary heart disease, regardless of age and sex, showed serum cholesterol or blood pressure levels in a range in which genetic influences almost certainly play an appreciable role. These figures may be compared with an over-all prevalence rate of 24 per cent for cholesterol and/or blood pressure elevations in the total Tecumseh population, including the persons with coronary disease, between ages 40 and 69 .

\section{Evolution and natural selection}

In the foregoing discussion, an attempt was made to show that the exact mechanisms and role of heredity in the development of coronary heart disease remain to be established. At the same time, the evidence certainly favors the belief that genetic factors are involved in the pathogenesis of the atherosclerotic disorders. Assuming the existence of such factors, we may ask whether the striking differences in the frequency of coronary heart disease among different population groups may have, in part, a genetic basis. Are some of the genes predisposing toward atherosclerosis more frequent, say, in the United States than in the Netherlands or Japan? There is clearly no definitive answer to this question at the present time, although there is some circumstantial evidence that these particular differences may be related more to environmental than genetic variations. Even in our study among Italian and Jewish clothing workers in New York City, ${ }^{41}$ specifically designed to answer some of these questions, we hesitated to ascribe the higher prevalence of coronary disease among the Jewish men necessarily to genetic factors, since there were potential environmental differences, such as early rather than current dietary habits and others, which remained under suspicion.

Regardless of the reasons for the geographic and ethnic differences mentioned, the fact remains that coronary disease is highly prevalent in some populations. If hereditary factors contribute significantly to this situation, it must follow that the responsible genes must also occur with considerable frequency. At least two factors may have contributed to this situation which causes concern from a biulogic point of view, unless one holds the cynical attitude that this state of affairs helps to eliminate older people who tend to be a burden on the young and vigorous. Firstly, if a disease is partly determined by heredity, and if age of onset is also under hereditary influences, natural selection will gradually determine that it will occur more and more in the postreproductive period ${ }^{49}$ - the time at which coronary disease actually becomes manifest most commonly. Secondly, it has been suggested that the genes predisposing to atherosclerosis may have a sclective advantage, for instance in times of fam in $e^{50,51}$ when the carriers of these traits might withstand the hazards of starvation by being able to store fat more effectively in their depots and, alas, their arteries.

Thus, natural selection could cause accumulation of these genes in the population. Could natural selection also have the reverse effect and help in eliminating these genes? Lown and Stare ${ }^{52}$ have stated that diseases such as atherosclerosis have not been culled from the stream of inheritance by rigorous evolutionary process, since natural selection cannot operate beyond the phase of reproduction. Although this is true in the strict sense, it is entirely conceivable that the carriers of these genes might be less fertile or show a higher mortality in the prereproductive or reproductive periods. Even small fertility or mortality differentials over the generations may have a profound effect on gene frequencies. ${ }^{53}$ The factors involved in causing fertility or mortality differentials may be affected by both biologic and sociocultural influences. Genetic and environmental interactions which determine selective pressures are well recognized. ${ }^{54,55}$ Without speculating on possible mechanism, it is merely stated that the diseases which present past the reproductive period are not necessarily removed from the forces of natural selection, and that, potentially, selective influences are not entirely powerless to counteract the accumulation of the responsible genes.

Even if there were an inexorable tendency for these genes to become more frequent, it would not necessarily follow, as McKeow $n^{56}$ has proposed, that only selective breeding is likely to result in a profound change in the causes and extent of 
mortality in the postreproductive period. If the genes which predispose toward atherosclerosis are sensitive to environmental influences, their expression might be appreciably suppressed by changes in the mode of life. The need, therefore, would be to detect the carriers of these traits early in life so that preventive measures might be instituted among susceptible persons in order to forestall the development of pathologic changes.

\section{Summary}

Currently available data on aggregations of coronary heart disease among relatives have been reviewed, and the interrelationships between genetic and environmental factors responsible for familial predispositions toward coronary atherosclerosis have been discussed. Although the evidence suggests a definite but not striking tendency for coronary disease to cluster in families, a quantitative assessment of the relative importance of familial influences in the genesis of these disorders is not possible at the present time. The first step loward the solution of the methodological problems involved lies in the recognition of their nature. It is suggested that the true extent of familial aggregations of coronary heart disease can only be estimated with assurance on the basis of long-term, rather than one-time, studies of families representative of the population at large.

\section{REFERENCES}

1. Katz, L. N., Levine, S. A., Page, I. H., Sprague, H. B., Stamler, J., Stare, F. J., White, P. D., and Wright, I. S.: A statement on arteriosclerosis, National Health Education Committee, Inc., 135 East 42nd St., New York 17, N. Y. (undated).

2. McKusick, V. A., and Murphy, E. A.: Genetic factors in the etiology of myocardial infarction. In James, T. N., and Keyes, J. W., editors: The etiology of myocardial infarction, Boston, 1963, Little, Brown and Company.

3. McKusick, V. A.: Genetic factors in cardiovascular diseases. I. The four major types of cardiovascular disease, Mod. Concepts Cardiovas. Dis. 28:535, 1959.

4. Epstein, F. H., Block, W. D., Hand, E. A., and Francis, T., Jr.: Familial hypercholesterolemia, xanthomatosis and coronary heart disease, Am. J. Med. 26:39, 1959.

5. Boas, E. P., Parets, A. D., and Adlersberg, D.: Hereditary disturbance of cholesterol metabolism: a factor in the genesis of atherosclerosis, Am. Heart J. 35:611, 1948.
6. Wilkinson, C. F., Jr., Hand, E. A., and Fliegelman, M. T.: Essential familial hypercholesterolemia, Ann. Int. Med. 29:671, 1948.

7. Dawber, T. R., and Kannel, W. B.: Susceptibility to coronary heart disease, Mod. Concepts Cardiovas. Dis. 30:671, 1961.

8. Keys, A.: The risk of coronary heart disease, (Editorial) Circulation 23:805, 1961.

9. White, P. D.: The importance of heredity in coronary heart disease, Circulation 22:296, 1960.

10. Epstein, F. H.: Epidemiology of coronary heart disease. In Jones, A. M., editor: Modern trends in cardiology, London, 1960, Butterworths.

11. Stamler, J.: Cardiovascular disease in the United States, Am. J. Cardiol. 10:319, 1962.

12. Gertler, M. M., and White, P. D.: Coronary heart disease in young adults: a multidisciplinary study, Cambridge, Mass., 1954, Harvard University Press.

13. Gertler, M. M.: Personal communication.

14. Cady, L. D., Jr., Gertler, M. M., Guttsch, L. G., and Woodbury, M. A.: The factor structure of variables concerned with coronary heart dis. ease, Behavioral Sc. 6:37, 1961.

15. Thomas, C. B., and Cohen, B. H.: The familial occurrence of hypertension and coronary artery disease, with observations concerning obesity and diabetes, Ann. Int. Med. 42:90, 1955.

16. Russek, H. I., and Zohman, B. L.: Relative significance of heredity, diet and occupational stress in coronary heart disease of young adults, Am. J. M. Sc. 235:266, 1958.

17. Shanoff, H. M., Little, A., Murphy, E. A., and Rykert, H. E.: Studies of male survivors of myocardial infarction due to "essential" atherosclerosis. I. Characteristics of the patients, Canad. M. A. J. 84:519, 1961.

18. Lew, E. A.: Some implications of mortality statistics relating to coronary artery disease, J. Chron. Dis. 6:192, 1957.

19. Harvald, B., and Hauge, M.: A catamnestic irvestigation of Danish twins, Acta genet. et statist. med, $8: 287,1958$.

20. Verschuer, O. v.: Die Zwillingsforschung im Dienste der inneren Medizin, Verhandl. Deutsch. Ges. inn. Med. 64:262, 1958.

21. Benedict, R. B.: Coronary heart disease in identical female twins, Am. J. Med. 24:814, 1958.

22. Lees, R. S., Canellos, G. P., Rosenberg, I. H., and Hatch, F. T.: Myocardial infarction in one of a pair of twenty-seven-year-old identical male twins, Am. J. Med. 34:741, 1963.

23. Clancy, R. E., Trulson, M. F., Hegsted, M., and Stare, F. J.: Comparisons of Irish-born Bostonians with their brothers living in Ireland. Presented at Conference on Cardiovascular Disease Epidemiology; American Heart Assn., Chicago Heart Assn., National Heart Institute, Chicago, III., February, 1963.

24. Ciocco, A.: Data on the concurrence of death from tuberculosis, influenza and pneumonia, cancer and heart disease among husbands and wives, Pub. Health Rep. 57:1333, 1942.

25. Dawber, 'T. R., Moore, F. E., and Mann, G. V.: Coronary heart disease in the Framingham 
study, Am. J. Pub. Health 47: Suppl. to No. 4., p. $4,1957$.

26. Epstein, F. H., and Kjelsberg, M. O.: Coronary heart disease in relation to blood pressure and cholesterol levels in population studies. Presented at conference on "Contributions of Genetics to Epidemiologic Studies of Chronic Diseases," Ann Arbor, Michigan, 1963, Am. J. Pub. Health (in press).

27. Cicchinelli, A. L.: The composite of two Gaussian distributions as a model for blood pressure distributions in man (doctoral dissertation), Ann Arbor, Michigan, 1962. University of Michigan Microfilm publication.

28. Johnson, B. C., Kjelsberg, M. O., Epstein, F. H., Payne, M. W., and Hayner, N. S.: Comparisons between blood pressure and cholesterol levels among family members. Presented at Conference on Cardiovascular Disease Epidemiology, Chicago, 1963 (to be published).

29. Miall, W. E., and Oldham, P. D.: The hereditary factor in arterial blood pressure, Brit. $\mathrm{M}$. J. $1: 75,1963$.

30. Schaefer, L. E., Adlersberg, D., and Steinberg, A. G.: Heredity, environment and serum cholesterol: a study of 201 families, Circulation $17: 537,1958$.

31. Thomas, C. B.: Observations on some possible precursors of essential hypertension and coronary artery disease. V. Hypercholesteremia in healthy young adults, Am. J. M. Sc. 232:389, 1956.

32. Basset, D. R.: Serum lipids in young males with parental atherosclerosis, Am. J. M. Sc. 243:740, 1962.

33. Gofman, J. W.: Coronary heart disease, Springfield, Ill., 1959, Charles C Thomas Publisher.

34. Pell, S., and D'Alonzo, C. A.: A three-year study of myocardial infarction in a large employed population, J.A.M.A. 175:463, 1961.

35. Brontë-Stewart, B., Botha, M. C., and Krut, L. H.: ABO blood groups in relation to ischaemic heart disease, Brit. M. J. 1:1646, 1962.

36. Brontë-Stewart, B., and Krut, L. H.: The interdependence of prospective and retrospective studies in research on ischaemic heart disease, J. Atheroscler. Res. 2:317, 1962.

37. Murphy, E. A., and Mustard, J. F.: Coagulation tests and platelet economy in atherosclerotic and control subjects, Circulation 25:114, 1962 .

38. Hirschhorn, $\mathrm{K}$, and Hirschhorn, R : Incidence of familial hyperlipemia, Science 129:716, 1959.
39. Boas, E. P.: Arteriosclerosis and diabetes, I. Mt. Sinai Hosp. 19:411, 1952.

40. Conn, J. W., and Fajans, S. S.: The prediabetic state. A concept of dynamic resistance to a genetic diabetogenic influence, Am. J. Med. $31: 839,1961$

41. Epstein, F. H., Boas, E. P., and Simpson, R.: The epidemiology of atherosclerosis among a random sample of clothing workers of different ethnic origins in New York City. I. Prevalence of atherosclerosis and some associated characteristics. II. Associations between manifest atherosclerosis, serum lipid levels, blood pressure, overweight and some other variables, $J$. Chron. Dis. 5:300, 329, 1957.

42. Boas, E. P., Simpson, R., Steinberg, A. G., and Epstein, F. H.: Unpublished observations.

43. Epstein, F. H., and Francis, T., Jr.: Unpublished observations.

44. Francis, T., Jr.: Aspects of the Tecumseh study, Pub. Health Rep. 76:963, 1961.

45. Epstein, F. H.: An epidemiological study in a total community: The Tecumseh project, Univ. of Mich. Med. Bull. 26:307, 1960.

46. Napier, J. A.: Field methods and response rates in the Tecumseh Community Health Study, Am. J. Pub. Health 52:208, 1962.

47. Fraser Roberts, J. A.: Multifactorial inheritance in relation to normal and abnormal human traits, Brit. M. Bull. 17:241, 1961.

48. Edwards, J. H.: The genetic basis of common diseases, Am. J. Med. 34:627, 1963.

49. Platt, R.: Life: biological, not biographical, Lancet $1: 61,1956$.

50. Moynahan, E. J.: Medieval famines and 20th century heart disease, (Letter to Editor) Lancet 1:673, 1961 .

51. McKusick, V. A.: Natural sclection and contemporary cardiovascular disease, (Editorial) Circulation $27: 161,1963$.

52. Lown, B., and Stare, F. J.: Atherosclerosis, infarction and nutrition, (Editorial) Circulation $20: 161,1959$.

53. Neel, I. V.: The study of natural selection in primitive and civilized human populations, Human Biol. 30:43, 1958.

54. Neel, J. V.: Medicine's genetic horizons, Ann. Int. Med. 49:472, 1958.

55. Dobzhansky, T.: Mankind evolving, New Haven, 1962, Yale University Press.

56. Mckeown, T.: Priorities in preventive medicine, New England J. Med. 264:594, 1961. 